\title{
Comparison of dietary and health behaviour by BMI in Male Employees of KOREA
}

\author{
H. R. Won ${ }^{1}$, S. G. Rhie ${ }^{2}$ and I. Y. Chang ${ }^{2}$ \\ ${ }^{1}$ Department of Food and Nutrition, Sangji University, Wonju, South Korea and ${ }^{2}$ Department of Food and Nutrition, The \\ University of Suwon, Hwaseong, South Korea
}

For increase of labour productivity and to prepare senile health, middle aged needed reduce the risk of chronic disease. A westernised diet and lack of exercise have contributed to increased morbidity and mortality by degenerative diseases ${ }^{(1)}$. The aim of this project was to know the structure of dietary and health behaviour changes for good health of middle aged employees ${ }^{(2)}$.

This study was investigated on dietary and health behaviour of office work employees in middle area (Seoul, Suwon, Chunan and Daejeon) of Korea. The subjects 239 answered the questions during Nov. 2009-Mar. 2010. The questionnaire was included health behaviours (drinking, smoking, exercise, weight concept) dietary behaviour (meal snack eat-out and nutrition knowledge and attitudes). By calculating BMI they were divided into three groups normal weight, overweight and obesity by the reference of WHO for oriental ${ }^{(3)}$. Results were analysed by the statistical program (SAS ver. 8.1), presented by frequencies and percentage. Dietary habits were scored by Likert scale five points and obtained the mean and standard deviation. Obtaining significant BMI group difference, Chi-square and GLM (General Linear model) used. The subjects composed the age of 20th $69.8 \%$, 30th $21.6 \%$ and 40 th $8.7 \%$. For all participants $62 \%$ not married, $26.8 \%$ received nutrition education by mass media (45.4\%). Overweight and obesity compared with normal weight group were drinking more frequently and the amount $(P<0.001)$. The smoking rates of overweight was more often than obese and normal weight group $(P<0.001)$. Forty-one percentage of the subjects had to exercise once or twice a week, but overweight $(54.4 \%)$, obesity $(40.5 \%)$ did not exercise at all $(P<0.01)$. Many of them $(67.8 \%)$ did not at all strength training, except $23.1 \%$ of normal weight group did $3 \mathrm{~d}$ a week $(P<0.05)$. Usual meal frequency was two times per day $(53.6 \%)$, there was significant difference in the number in each group (normal weight $(67.7 \%)$ overweight $(56.7 \%)$ obesity group $(50 \%)(P<0.001)$. Half $(48.5 \%)$ of them did not eat snacks, but $1-2$ servings a day in obese $(27.4 \%)$ and normal $(33.9 \%)(P<0.001)$. Food service frequency was higher in obese group $(P<0.001)$. The intake of overall foods and meats were higher in obese group than in the normal group $(P<0.001)$. Eating habits showed overall bad scores, but nutrition knowledge was more in obese group. Nutrient supplements, health food or herbal medicine taking were more frequent in normal group $(P<0.001)$. Vitamin supplement was selected rather than replace when poor meal in obese group $(P<0.001)$. Drinking, smoking and fitness were affected in obesity of men employees and nutrition knowledge, attitude, behaviour were deemed to be of a very low status. To learn and practice of proper nutritional health management would need for healthy employees delay and prevention of chronic degenerative diseases comes as obesity. These data suggest that the need for a nutrition education system is most urgent for male employees.

1. Sakamoto M, Chiu P, Chen CM et al. (1995) Proc 7th CAN, pp. 54-65.

2. Molarius A (2003) Eur J Epidemiol 18, 227-234.

3. WHO (1997) Obesity preventing and the global epidemic of a WHO consultation on obesity. 